\title{
Special issue on swarm intelligence and its applications to engineering. Journal: evolutionary intelligence
}

\author{
Jagdish Chand Bansal ${ }^{1} \cdot$ Kusum Deep ${ }^{2} \cdot$ Atulya K. Nagar $^{3}$
}

Published online: 3 August 2021

(c) The Author(s), under exclusive licence to Springer-Verlag GmbH Germany, part of Springer Nature 2021

Over the last 25 years, swarm intelligence has shown its potential in solving complex engineering optimization problems. This special issue of the journal Evolutionary Intelligence is a vast collection of research articles in the field of swarm intelligence and its applications to engineering. An open Call for Paper was issued for this special issue. The guest editors feel privileged to produce this special issue of one of the well regarded and reputed journal Evolutionary Intelligence.

From a wide range of interesting research papers on various aspects of swarm intelligence, the editors carefully selected 15 papers through rigorous peer-review process which has been the basis for the final and considerate decisions in the inclusion of these articles. Key strengthens of these articles are summarized below.

In the first article, Senthilnath et al. solved the data clustering problem using flower pollination algorithm (FPA). The results are shown to be better than many other nature inspired algorithms. In the second article by Shukla and Kanungo a new Grey Wolf Optimizer variant, Sigmoidal Grey Wolf Optimization (SGWO) is presented and applied to enhanced $\mathrm{BOF}$ framework for clustering of features. The next article, 'An Efficient Hybrid PSO Polygamous Crossover Based Clustering Algorithm' by Sharma and Chhabra presents a hybrid metaheuristic approach PSOPC that depends on the integrated concept of PSO, genetic algorithm and the polygamous concept for mating. The proposed

Jagdish Chand Bansal

jcbansal@sau.ac.in

Kusum Deep

kusumfma@iitr.ernet.in

Atulya K. Nagar

atulya.nagar@hope.ac.uk

1 South Asian University, Delhi, India

2 Indian Institute of Technology (IIT), Roorkee, India

3 Liverpool Hope University, Liverpool, UK algorithm is applied to seven real clustering datasets. Results are compared with several other meta-heuristics. Authors Bairathi and Gopalani in their article, 'Numerical Optimization and Feed-Forward Neural Networks Training using an Improved Optimization Algorithm: Multiple Leader Salp Swarm Algorithm' presents the concept of multiple leader in salp swarm algorithm (SSA) including a new variant MLSSA. The authors have also applied MLSSA for neural network training. The next article, 'Multi-objective three level parallel PSO algorithm for structural alignment of complex RNA sequences' by Lalwani and Sharma proposes a caching enabled parallel multi-objective tri-level particle swarm optimization algorithm (MO-3LPPSO) with objective to address a problem from bioinformatics, structural alignment of complex RNA sequences. Khandelwal et al. in their article "Voltage stability constrained transmission network expansion planning using fast convergent grey wolf optimization algorithm' proposed a GWO variant namely, fast convergent GWO (FCGWO) and applied it to solve Transmission network expansion planning problem (TNEP) and voltage stability constrained TNEP (VSC-TNEP) for IEEE-24 bus test system. The next article, 'Improved Gbest Artificial Bee Colony Algorithm for the constraints optimization problems' by Sharma et al. presents a new variant of Gbest-guided Artificial Bee Colony (ABC) algorithm by updating employed and onlooker bee phases. This enhances the efficiency of Gbest-guided ABC. The proposed variant has also shown to be effective to avoid the stagnation. Tiwari and Jain introduced a new adaptive Jaya Algorithm in their article, 'An optimal feature selection method for histopathology tissue image classification using jaya algorithm'. The proposed adaptive jaya algorithm modifies the update equation using the best and the worst solutions. The proposed modified algorithm has been applied for feature selection to classify the histopathological images into epithelium, connective tissue, muscular tissue, and nervous tissue classes. The article 'An image segmentation method using logarithmic kbest gravitational search algorithm based 
superpixel clustering' by Mittal and Saraswat introduces a new superpixel-based clustering method to efficiently perform the image segmentation. The superpixels are further clustered into the required number of regions by a newly proposed variant of gravitational search algorithm namely, logarithmic kbest gravitational search algorithm. Authors Shekhawat et al. in their paper, 'Twitter Sentiment analysis using hybrid Spider Monkey Optimization Method' proposed an innovative hybrid clustering approach, namely the spider monkey optimization algorithm using k-means (SMOK) for examining the sentiments of different tweets. In the proposed mechanism, the results obtained from $\mathrm{k}$-means are used to initialize the population intelligently in SMO. The article. 'Random Offset Minimization in Low Frequency Front-End Amplifiers using Swarm Intelligence based techniques' introduces a new computationally efficient approach for minimizing the random offset voltages in front end neural recording or biomedical amplifiers using swarmbased algorithms. The next article. 'Improved Convolutional Neural Network based Histopathological Image Classification' by Rachapudi and Devi presents a new architecture for the convolutional neural network for the classification of the histopathological images. The proposed network is defined with multiple combination of convolutional layer, activation layer, max-pooling layer, drop-out layer and dense layer. Sharma et al. in their article, 'Limacon inspired artificial bee colony algorithm for numerical optimization' proposed a new variant of $\mathrm{ABC}$ by incorporating local search inspired by the limacon curve. The next article 'Lunar Cycle Inspired PSO for Single Machine Total Weighted Tardiness Scheduling Problem' by Gupta et al. proposes an efficient variant of the PSO algorithm by incorporating lunar cycle inspired local search in PSO. The proposed algorithm has also been applied to Single machine total weighted tardiness scheduling problem. In the last article, 'An efficient nuclei segmentation method based on roulette wheel whale optimization and fuzzy clustering' by Vishnoi et al. a new variant of the whale optimization method, namely RSWOA is introduced for the automated segmentation of nuclei in histopathological images. The proposed variant employs roulette wheel selection for electing the search agents instead of selecting randomly for exploring the entire search space.

An important aim of this special issue has been to make available recent research in the field of swarm intelligence and its applications to various engineering optimization problems. We hope that students, researchers, and academics working in the field of swarm intelligence will benefit from the research presented in this volume.

As guest editors, we would like to express our deep sense of gratitude to the Editors-in-Chief, Prof. Vincenzo Loia, for providing us with the opportunity to host this special issue of the journal Evolutionary Intelligence. We also thank the authors for their contributions, including those whose papers were not included. We are also thankful to many reviewers whose meaningful and considerate feedback as well as comments made enhanced the quality of these papers for inclusion in this special issue. We also express our gratitude for the editorial staff at Springer Nature for the support received during the producing this collection.

Publisher's Note Springer Nature remains neutral with regard to jurisdictional claims in published maps and institutional affiliations. 